\title{
Nanostructured Polypyrrole Powder: A Structural and Morphological Characterization
}

\author{
Edgar A. Sanches, ${ }^{1}$ Scarlat F. Alves, ${ }^{1}$ Juliana C. Soares, ${ }^{2}$ Arnaldo M. da Silva, \\ Cristina G. da Silva, ${ }^{1}$ Sérgio M. de Souza, ${ }^{1}$ and Hidembergue O. da Frota ${ }^{1}$ \\ ${ }^{1}$ Departamento de Física (DF), Universidade Federal do Amazonas (UFAM), 69077-000 Manaus, AM, Brazil \\ ${ }^{2}$ Universidade de São Paulo (USP), Instituto de Física de São Carlos (IFSC), 13566-590 São Carlos, SP, Brazil
}

Correspondence should be addressed to Edgar A. Sanches; sanches.ufam@gmail.com

Received 28 April 2015; Accepted 28 July 2015

Academic Editor: Mohamed Bououdina

Copyright (C) 2015 Edgar A. Sanches et al. This is an open access article distributed under the Creative Commons Attribution License, which permits unrestricted use, distribution, and reproduction in any medium, provided the original work is properly cited.

\begin{abstract}
Polypyrrole (PPY) powder was chemically synthesized using ferric chloride $\left(\mathrm{FeCl}_{3}\right)$ and characterized by X-ray diffraction (XRD), Le Bail Method, Fourier Transform Infrared Spectrometry (FTIR), and Scanning Electron Microscopy (SEM). XRD pattern showed a broad scattering of a semicrystalline structure composed of main broad peaks centered at $2 \theta=11.4^{\circ}, 22.1^{\circ}$, and $43.3^{\circ}$. Crystallinity percentage was estimated by the ratio between the sums of the peak areas to the area of amorphous broad halo due to the amorphous phase and showed that PPY has around 20 (1)\%. FTIR analysis allowed assigning characteristic absorption bands in the structure of PPY. SEM showed micrometric particles of varying sizes with morphologies similar to cauliflower. Crystal data (monoclinic, space group P 21/c, $a=7.1499$ (2) $\AA, b=13.9470$ (2) $\AA, c=17.3316$ (2) $\AA, \alpha=90 \AA, \beta=61.5640$ (2) $\AA$ and $\gamma=90 \AA$ ) were obtained using the FullProf package program under the conditions of the method proposed by Le Bail. Molecular relaxation was performed using the density functional theory (DFT) and suggests that tetramer polymer chains are arranged along the " $c$ " direction. Average crystallite size was found in the range of 20 (1) $\AA$. A value of $9.33 \times 10^{-9} \mathrm{~S} / \mathrm{cm}$ was found for PPY conductivity.
\end{abstract}

\section{Introduction}

Intrinsically Conductive Polymers (ICPs) with conjugated double bonds have attracted attention in fundamental and applied researches, emerging with great deal of technological applications [1-5]. Polypyrrole, polythiophene, polyaniline, and polyacetylene have been intensively studied due to their good environmental stability and high electrical conductivity, making them more desirable than metals in many cases $[6,7]$.

Among ICPs, polypyrrole (PPY) is especially promising in commercial applications [8]. Polypyrrole has been applied as electrochromic and electronic devices, membrane separation, light-weight batteries, sensors, rechargeable batteries, microwave shielding, supercapacitors, drug delivery, corrosion protection, and artificial muscle [1,9-15]. However, the inherently poor solubility in common solvents, which originates from the strong inter- and intrachain interactions, has limited some PPY's practical applications [16-19]. Figure 1 shows the PPY molecular structure.
Many studies have been made to characterize PPY, but few focus on structural characterization through structural refinement methods. Understanding the structure and morphology of semicrystalline materials is essential to the development of new technological applications. X-ray diffraction (XRD) pattern was used to obtain structural information using the Le Bail whole powder pattern decomposition method applied to a semicrystalline PPY for determination of cell parameters and crystallite size and shape. Fourier Transform Infrared Spectroscopy (FTIR) was used for structural information of the bonds; Scanning Electron Microscopy (SEM) was carried out for the investigation of the polymer morphology. Then, these results were correlated with the obtained electrical conductivity.

\section{Experimental}

2.1. Polymer Synthesis. Pyrrole (Sigma-Aldrich) was distilled before use. All other reagents were used as received. All 
<smiles>[3H][Y]1c(-c2ccc(C(C)(C)C)[nH]2)ccc1-c1ccc(C(C)(C)C)[nH]1</smiles>

FIgURe 1: Polypyrrole molecular structure.

reactions were conducted at room temperature. Chemically polymerized polypyrrole was obtained by oxidative polymerization using $\mathrm{FeCl}_{3}$ as oxidant in aqueous medium based on the literature [20], with some modifications. Pyrrole (1.0 M) solution was added drop by drop to the oxidizing agent aqueous solution in the ratio of $1: 3$. Then, the polymerization was conducted for 3 hours under constant stirring using $300 \mathrm{rpm}$. This preparation was filtered under vacuum and washed with distilled water and methanol.

2.2. Fourier Transform Infrared Spectroscopy (FTIR). FTIR spectra were measured in a spectrophotometer Bomem-MB Series, Hartmann \& Braun, in the range of $400-2000 \mathrm{~cm}^{-1}$ and 16 scans. Pellets were prepared with $\mathrm{KBr}$ in mass ratio of $1: 100$.

2.3. XRD Measurements and Crystallinity Estimative. XRD data were obtained at the laboratory of X-ray crystallography of IFSC/USP, São Carlos, SP, Brazil, using a Rigaku Rotaflex diffractometer equipped with graphite monochromator and rotating anode tube, operating with $\mathrm{Cu} \mathrm{K}_{\alpha}, 50 \mathrm{kV}$, and $100 \mathrm{~mA}$. Powder diffraction patterns were obtained in step scanning mode, $2 \theta=5-70^{\circ}$, step of $0.02^{\circ}$ and $3 \mathrm{~s} / \mathrm{step}$, where $\theta$ is the Bragg angle. Routine software was used for the peak deconvolution of the semicrystalline pattern and determination of area due to the noncrystalline phase. Crystallinity percentage was estimated by the ratio between the sums of the peak areas to the area of noncrystalline broad halo due to the noncrystalline phase.

2.4. Le Bail Method. Le Bail Method was performed using the software package FullProf [21]. All parameters were refined by the least-squares method [22]. The pseudo-Voigt function modified by Thompson-Cox-Hastings was used as peak profile function [23]. Instrumental resolution function parameters were obtained from a lanthanum hexaborate standard, $\mathrm{LaB}_{6}$. The PPY structural parameters obtained by Warren and Madden [11] were used as initial parameters for the Le Bail Method (monoclinic, $a=13.40 \AA, b=$ $7.30 \AA, c=3.75 \AA, \alpha=65^{\circ}, \beta=90^{\circ}$, and $\gamma=90^{\circ}$ ). Spherical harmonics parametrization (SHP) [24] was used to account for the possible crystallite anisotropy; particle size was determined from the information on line profile (peak width and shape) $[25,26]$.

2.5. Geometry Optimization. Molecular relaxation was performed using the density functional theory (DFT) as implemented in Quantum Espresso package [27], with the generalized gradient approximation (GGA) of Perdew-BurkeErnzerhof (PBE) for the exchange-correlation energy [28] and the BFGS quasi-Newton algorithm for the geometry

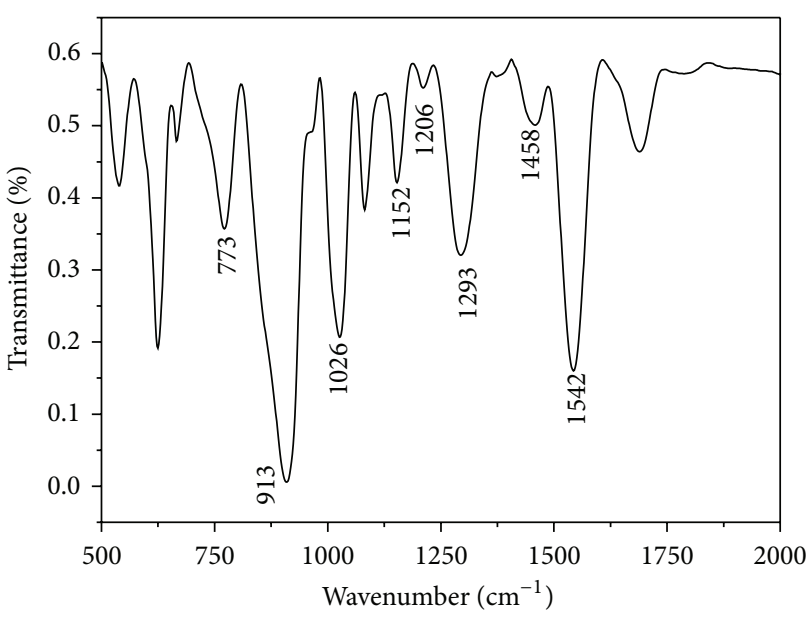

Figure 2: FTIR of polypyrrole.

optimization. The energy cutoff for the wave functions and for the charge density was taken as $0.816 \mathrm{keV}$ and $3.264 \mathrm{keV}$, respectively. The optimization process was evaluated until the forces on each atom become less than $10^{-4} \mathrm{eV} / \AA$. The molecular plots were made with the XCrySDen package [29].

2.6. SEM Analysis. SEM experiments were performed using a Supra 35, Carl Zeiss, $3.0 \mathrm{kV}$. Powder samples were deposited on a carbon tape and the surface morphology was obtained at room temperature.

2.7. DC Electrical Conductivity Measurements. Electrical connections were made in PPY pellet which was coated with silver ink. Measurements were performed at room temperature $(300 \mathrm{~K})$ using a Keithley Model 2612A from $500 \mathrm{mV}$ to $10 \mathrm{~V}$.

\section{Results and Discussion}

3.1. FTIR Analysis. Figure 2 shows the FTIR spectra of pure PPY obtained from 2000 to $500 \mathrm{~cm}^{-1}$, the most useful range for chemical characterization of organic materials [30, 31]. Bands located at $773 \mathrm{~cm}^{-1}$ and $913 \mathrm{~cm}^{-1}$ were attributed to $\mathrm{C}-\mathrm{H}$ stretching. A band at $1026 \mathrm{~cm}^{-1}$ may be assigned to $=\mathrm{C}-\mathrm{H}$ in plane deformation vibration. A band at $1152 \mathrm{~cm}^{-1}$ was attributed to $\mathrm{C}-\mathrm{H}$ in and out of plane deformations. Bands at $1458 \mathrm{~cm}^{-1}$ and $1542 \mathrm{~cm}^{-1}$ were assigned to vibration of pyrrole ring $(\mathrm{C}-\mathrm{N})$ and ring stretching mode $(\mathrm{C}=\mathrm{C}, \mathrm{C}-$ C), respectively. Bands located at $1206 \mathrm{~cm}^{-1}$ and $1293 \mathrm{~cm}^{-1}$ were assigned, respectively, to $\mathrm{N}-\mathrm{C}$ stretching band and $=\mathrm{C}-$ $\mathrm{H}$ in plane vibration. Bands observed in the present study are consistent with those available in the scientific literature and confirmed the polymerization of pyrrole [32-37].

3.2. X-Ray Diffraction and Crystallinity Estimative. Structural aspects in polymers continue to be an interesting researched topic $[38,39]$. Understanding the regular arrangement of polymeric materials is essential for the evaluation of the material properties and the proposition of new technological applications. 


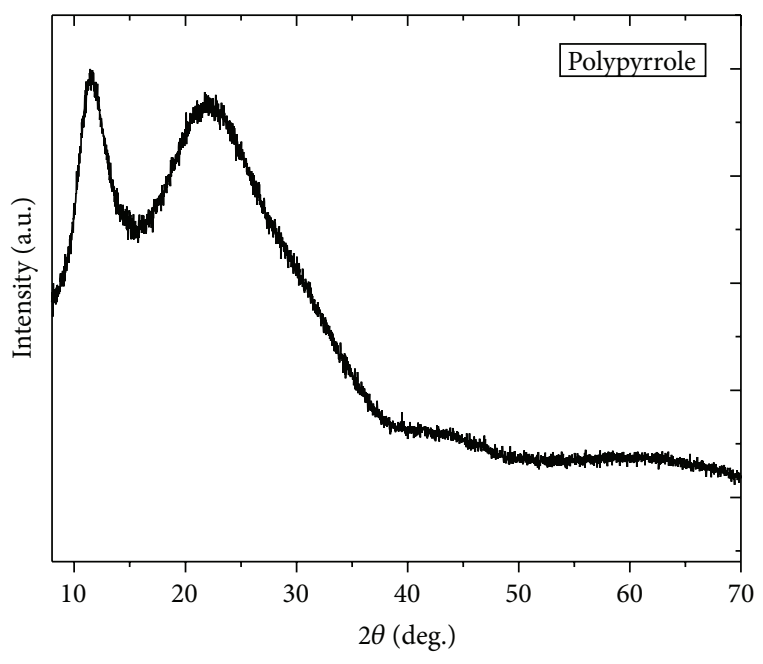

FIGURE 3: XRD of polypyrrole.

A typical X-ray diffraction pattern of PPY prepared using $\mathrm{FeCl}_{3}$ is presented in Figure 3 showing the position in $2 \theta$ of all possible Bragg reflections. Broad peaks were observed indicating some structural ordering of nanometric dimensions, typical of nanostructured polymers with low degree of crystallinity (fringed micelles model). XRD pattern showed that four broad peaks (better observed in Figure 4) related to the scattering from PPY chains at the interplanar spacing are centered at $2 \theta \sim 11.7^{\circ} ; 22.7^{\circ} ; 29.8^{\circ}$; and $44.3^{\circ}$ [40]. The large amount of disorder inherent in the PPY matrix means the X-ray peaks are broad Gaussians and the higher order peaks are severely damped [11].

To estimate the crystallinity percentage of semicrystalline polymers, it is assumed that the polymer consists of a welldefined mixture of noncrystalline and crystalline regions [41, 42]. Many methods have been applied for the estimation of crystallinity percentage in semicrystalline materials, and it has become increasingly apparent that these methods do not always give the same result. However, all current methods assume implicitly a two-phase model of crystallites embedded in a noncrystalline matrix.

The most used method to access the crystalline portion of a given material using XRD date is the deconvolution method [43]. For the curve fitting, assumptions such as the shape and number of peaks and an appropriate function have to be made. An important hypothesis for this analysis is that increased noncrystalline contribution is the main contributor to peak broadening. However, in addition to crystalline disorder (noncrystalline content), there are other intrinsic factors that influence peak broadening, such as crystallite size and nonuniform crystal strain.

Deconvolution of the XRD peaks is showed in Figure 4. The green curve, below the experimental black curve, corresponds to a Chebyshev polynomial [44] of sixth order, arranged through the minimum of the narrower peaks. The noncrystalline broad halo component (from $50^{\circ}$ to $70^{\circ}$ degrees in $2 \theta$ ) contributes to the XRD pattern with a broad halo generating diffuse scattering, which is often confused

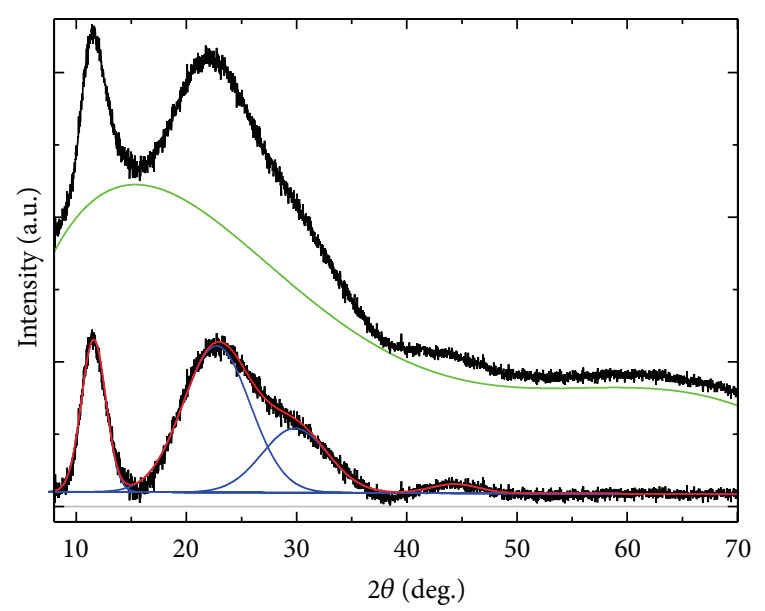

Figure 4: Peaks deconvolution of polypyrrole.

with the background. This broad halo shows a short-range structure, such as distance distributions between nearest neighbors. The resulting curve indicates a noncrystalline phase with a maximum at $2 \theta \sim 15.7^{\circ}$. Applying the Ehrenfest formula [45], $2 r \sin \theta=1.23 \lambda$, the interatomic distances $r$ can be estimated, where $\lambda$ is the wavelength of X-rays and $\theta$ is the half angle of diffraction. Thus, a wide distribution of distances with maximum at $6.9 \AA$ was noted. This is in agreement with the distances between nitrogen atoms of parallel chains, which correspond to $6.2 \AA$ [46].

Assuming the amorphous contribution, the Chebyshev polynomial is subtracted from the total pattern allowing the crystalline peaks obtainment, as shown in Figure 4. Comparing the experimental area with the area of the polynomial curve, the estimate of crystallinity percentage of PPY corresponds to $20 \%$. The four observed peaks were best fitted using Gaussian curves, revealing symmetrical features of the pattern, which is typical of isotropic structures.

3.3. Le Bail Method Analysis. The use of Le Bail Method $[47,48]$ to obtain structural information from semicrystalline patterns is not very common due to the large overlapped peaks on diffractograms. Nevertheless, it has been used to characterize some conducting polymers as polyaniline and substituted polyanilines [49-51]. This process uses iteratively the Rietveld decomposition formula for whole powder pattern decomposition (WPPD) in the FullProf program package [52]. A fair approximation to the observed integrated intensity can be made by separating the peaks according to the calculated values of the integrated intensities as follows:

$$
I_{k(\mathrm{obs})}=\sum_{j}\left\{\frac{w_{j \cdot k} \cdot S_{k(\mathrm{calc})}^{2} \cdot y_{j(\mathrm{obs})}}{y_{i(\mathrm{calc})}}\right\},
$$

where $w_{j \cdot k}$ is a measure of the contribution of the Bragg peak at position $2 \theta_{k}$ to the diffraction profile $y_{j}$ at position $2 \theta_{j}$. The sum is overall $y_{j(\mathrm{obs})}$, which can theoretically contribute to the integrated intensity $I_{k(\mathrm{obs})}$. So there is a bias introduced here by the apportioning according to the calculated intensities; this is why the observed intensities are in fact said to be 
TABLE 1: Le Bail Method applied for the structural analysis of PPY using the FullProf program: cell parameters, cell volume, average size and anisotropy, crystallite apparent size, and agreement factors.

\begin{tabular}{|c|c|}
\hline Refined parameters & PPY \\
\hline$a(\AA)$ & 7.1499 \\
\hline$b(\AA)$ & 13.9470 \\
\hline$c(\AA)$ & 17.3316 \\
\hline$\alpha\left(^{\circ}\right)$ & 61.5640 \\
\hline$\beta\left(^{\circ}\right)$ & 90.0000 \\
\hline$\gamma\left({ }^{\circ}\right)$ & 90.0000 \\
\hline$V\left(\AA^{3}\right)$ & 801.20 \\
\hline Global average size (anisotropy) $(\AA)$ & $20(3)$ \\
\hline Crystallite apparent size $_{100}(\AA)$ & 21 \\
\hline Crystallite apparent size $_{010}(\AA)$ & 15 \\
\hline Crystallite apparent size $_{001}(\AA)$ & 18 \\
\hline$R_{\mathrm{wp}}(\%)$ & 2.74 \\
\hline$R_{p}(\%)$ & 2.16 \\
\hline
\end{tabular}

"observed" under quotes, in the Rietveld method. These "observed" intensities are used in the $R_{B}$ and $R_{F}$ calculations [47].

The Le Bail Method allows only refining the unit cell parameters and what would be the effectively measured $S_{k(\text { calc })}$ intensities are only the intensities suggested by the profile fitting based on a distribution of intensities using a profile function [23] for each overlapped reflection constituting the few very broad peaks. These are due to the low crystallinity and small crystallite size.

This refining method has recently been utilized to obtain structural information of semicrystalline materials, even taking into account the large overlapped peaks on XRD patterns. Structural refinement of PPY through Le Bail Method started using as initial parameters those reported by Warren and Madden [11]. XRD patterns showed a good fit using a monoclinic unit cell model, P21/c, with lattices $a, b$, and $c$ equal to 7.1499 (2) $\AA, 13.9470$ (2) $\AA$, and 17.3316 (2) $\AA$, respectively, and the angle $\alpha$ equal to $61.5640(2)^{\circ}$. The observed $\left(I_{\text {obs }}\right)$ and calculated $\left(I_{\text {calc }}\right)$ diffractograms and the residual line $\left(I_{\text {obs }}-I_{\text {calc }}\right)$ as well as indexes for the main reflections are showed in Figure 5. Table 1 shows the refined parameters for PPY.

Anisotropic size broadening can be written as a linear combination of spherical harmonics and it is supposed that anisotropic size contributes only to the Lorentzian component of the total Voigt function. The explicit formula of the intrinsic integral breadth using the SPH treatment of size broadening is given by [26]

$$
\beta_{h}=\frac{\lambda}{D_{h} \cos \theta}=\frac{\lambda}{\cos \theta} \sum_{\operatorname{lmp}} a_{\mathrm{lmp}} y_{\mathrm{lmp}}\left(\Theta_{h} \Phi_{h}\right),
$$

where $\beta_{h}$ is the size contribution to the integral breadth of reflection $(h k l), y_{\operatorname{lmp}}\left(\Theta_{h} \Phi_{h}\right)$ are real spherical harmonics (arguments $\Theta_{h}$ and $\Phi_{h}$ are the polar angles of the vector $h$ with respect to a Cartesian crystallographic frame) [53], and $a_{\operatorname{lmp}}$ are refinable coefficients, depending on the Laue

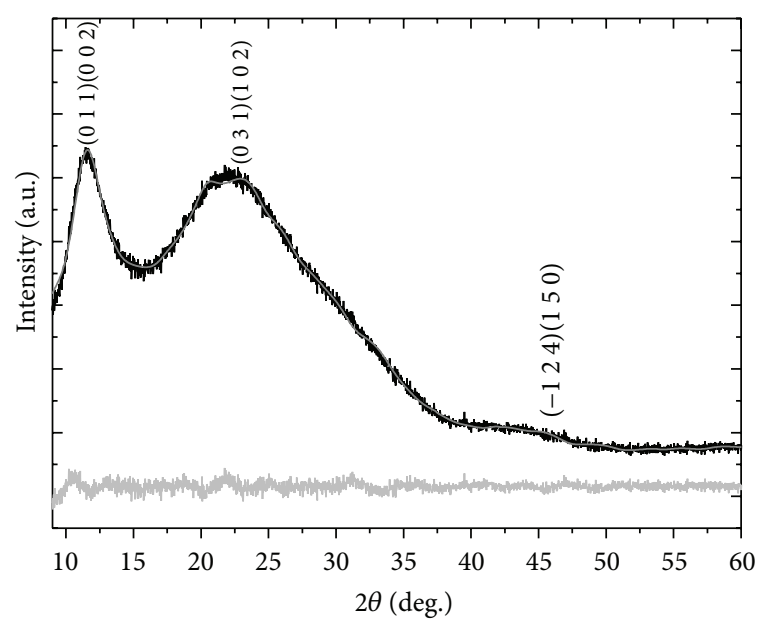

FIGURE 5: Le Bail Method applied to polypyrrole showing the main reflexions.

class. After refinement of the coefficients $a_{\mathrm{lmp}}$, the program calculates the apparent size $\left(D_{h}\right.$, in $\AA$ ) along each reciprocal lattice vector if the parameters $(U, V, W)_{\text {instr. }}$ are fetched to the program from an external instrumental resolution function file.

It was possible to visualize the crystallites in directions [100], [010], and [001] using the GFourier Program [54]. It is important to stress that these crystallite shapes do not refer to the actual shape but to the features of a rather rough and approximated model. Those shapes are related to the symmetry properties of the distribution of columns of scattering centers in crystalline domains of the given phase. PPY showed an average crystallite size of 20 (1) $\AA$ with a standard deviation (anisotropy) of $3 \AA$. It is important to stress that the standard deviation appearing in the global average apparent size is calculated using the reciprocal lattice directions so it is a measure of the degree of anisotropy, not of the estimated error. There is a smaller apparent size of $15 \AA$ in the [010] direction and almost equivalent along [100] and [001], respectively, 21 and $18 \AA$. The refined average crystallite size $2 \mathrm{D}$ and $3 \mathrm{D}$ projections for PPY are showed in Figures 6(a) and 6(b). Crystallite shape is quite similar with the particle morphology of PPY, which can be described as a cauliflowerlike shape morphology, as will be evidenced by SEM [55-57].

3.4. Geometry Optimization. In Figure 7 we present the molecular structure obtained after geometry optimization, where the monomers lie in different plans. Note that this figure is a model for PPY tetramer unit cell using the refined cell parameters obtained previously by the Le Bail Method. The chloride ions (counter ions) on the structure voids of PPY tetramer were not introduced in this model. This model suggests that polymer chains are arranged along the " $c$ " direction. For the same monomer, the interatomic distances between two $\mathrm{C}$ atoms or between a $\mathrm{C}$ atom and a $\mathrm{N}$ atom are slightly different: $1.37 \AA$ for N-C bond, $1.39 \AA$ for $\mathrm{C}-\mathrm{C}$ bond adjacent to the $\mathrm{N}-\mathrm{C}$ bond, and $1.41 \AA$ for $\mathrm{C}-\mathrm{C}$ bond opposite to the $\mathrm{N}$ atom. The $\mathrm{H}-\mathrm{N}$ and $\mathrm{H}-\mathrm{C}$ bond lengths are $1.01 \AA$ and 

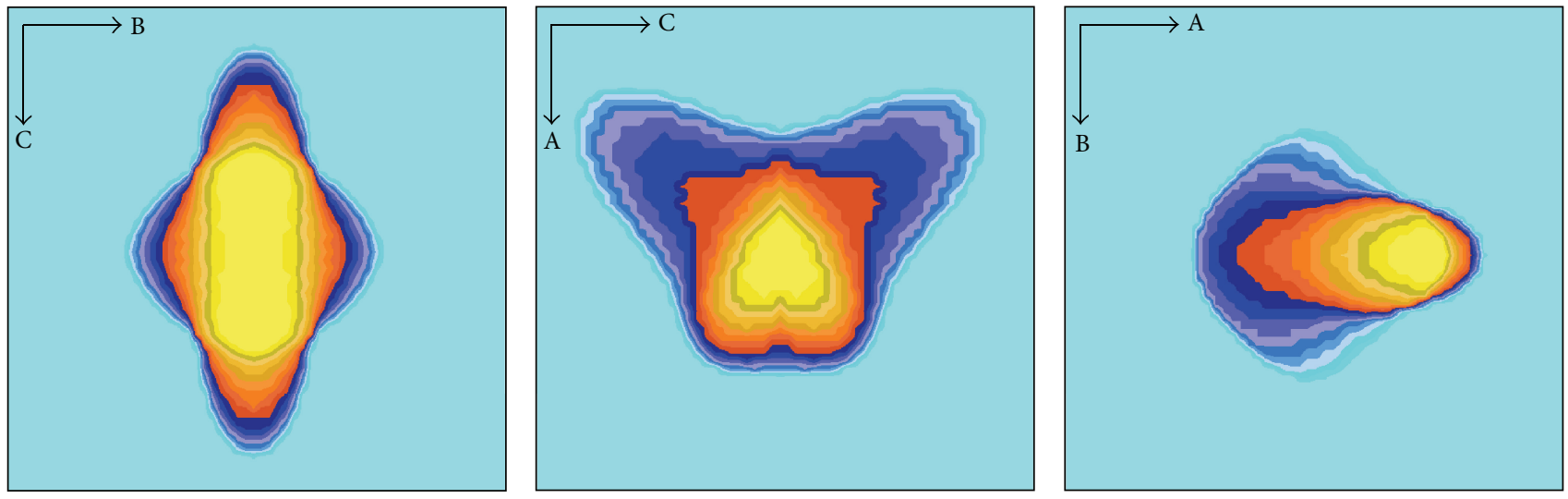

(a)
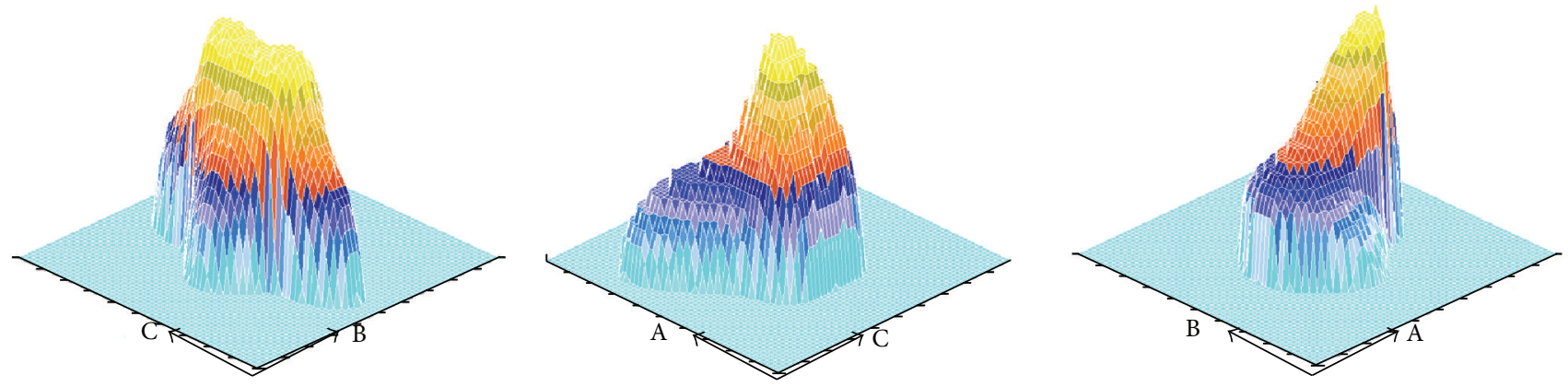

(b)

FIGURE 6: (a) 2D and (b) 3D average crystallite shape of PPY.

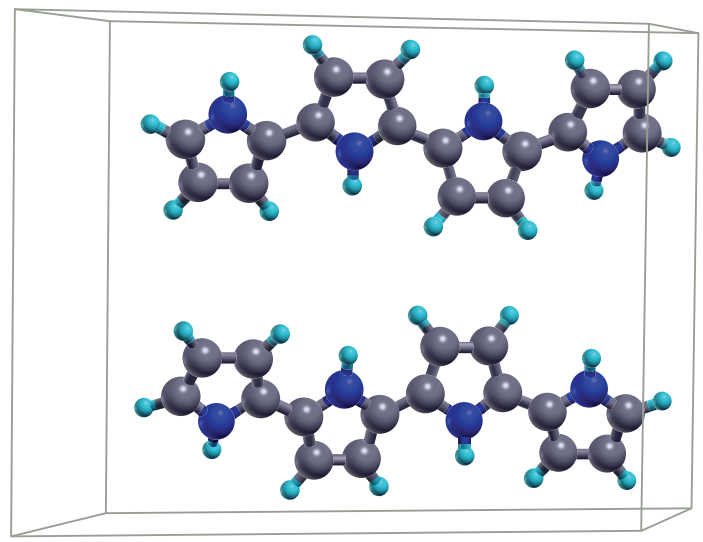

$\mathrm{C}$
$\mathrm{N}$
$\mathrm{H}$

FIGURE 7: Geometry optimization of PPY. 


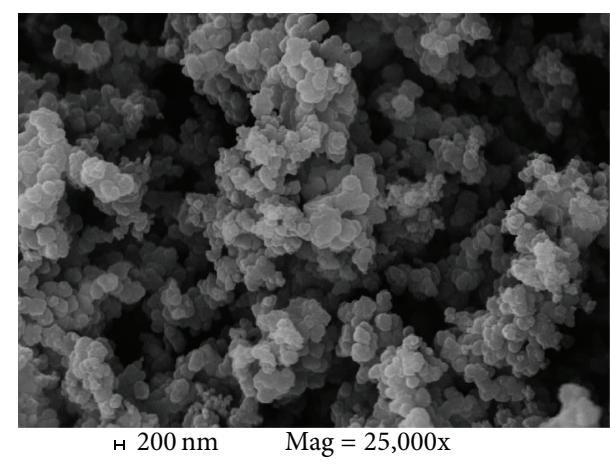

(a)

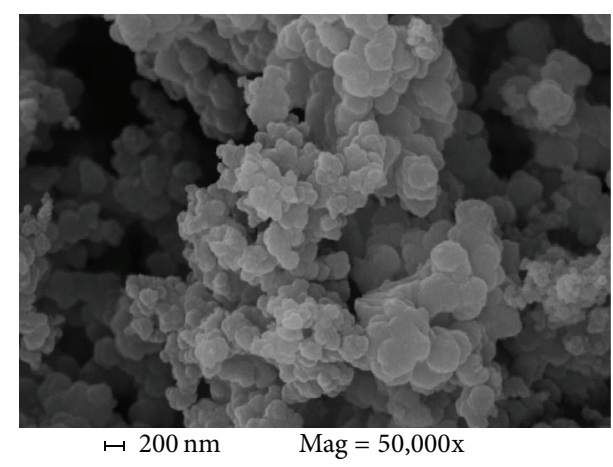

(b)

Figure 8: SEM images of PPY with increase of (a) 25,000 and (b) 50,000 times.

$1.08 \AA$, respectively. The bond length of two carbon atoms of adjacent monomers is around $1.44 \AA$.

3.5. SEM Analysis. SEM technique was used in order to identify the PPY morphology at room temperature. The morphological features of chemically synthesized PPY have revealed that mostly the polymeric growth is similar to the crystallite shapes. SEM images of PPY are shown in Figures 8(a) and 8(b). All images showed a cauliflower-like morphology constituted by microspherical grains [57]. It has been reported that this morphology is related to the dopant intercalation difficulty in the disordered polymeric chain $[56,57]$.

3.6. DC Electrical Conductivity Measurements. In the case of conducting polymers, the total conductivity is a function of interchain and intrachain mobility of the counter ion. The interchain mobility depends on the degree of crystallinity, temperature, and protonation (doping) level. In addition, the counter ions are capable of introducing defects by causing significant charge polarization in neighboring chains [58].

The relationship between applied voltage and current is given by $V=R I$, where $R$, the factor of proportionality, is the nanocomposite electrical resistance $(\Omega)$. To verify the DC electrical conductivity of nanocomposite, the dimensions of pellet sample were considered, represented by $R=\rho(l / A)$, where $l$ is the pellet thickness, $A$ is the area of the straight section, and $\rho$ is the electrical resistivity. For a plot of $I \times V$, the angular coefficient provides the inverse of resistivity, that is, electrical conductivity [58]:

$$
\sigma=\frac{1}{\rho}=\frac{l}{R A} .
$$

The DC electrical conductivity, $\sigma$, for pure PPY shows different values in the literature, closely related to their methods of synthesis. The DC electrical conductivity of PPY at room temperature was found to be $9.33 \times 10^{-9} \mathrm{~S} / \mathrm{cm}$. This value is of the same order of magnitude of the camphor sulfonic acid (CSA) doped PPY films obtained by Navale et al. (2014) [1].

\section{Conclusion}

We successfully synthesized PPY by chemical polymerization using iron (III) chloride as oxidant agent. Through FTIR technique, it was possible to identify characteristic absorption bands related to chemical bonds and functional groups present in PPY structure, which allowed its structural and organic chemical characterization. XRD technique showed a semicrystalline pattern with crystallinity around 20\%. PPY unit cell parameters were obtained through Le Bail Method. Microstructure analysis showed an average crystallite size around $20 \AA$. Molecular relaxation using the density functional theory (DFT) suggested that the tetrameric chain is lying along " $c$ " axis in unit cell. SEM analysis suggested a cauliflower-like morphology. DC electrical conductivity obtained at room temperature was found in the magnitude of $10^{-9} \mathrm{~S} / \mathrm{cm}$.

\section{Conflict of Interests}

The authors declare that there is no conflict of interests regarding the publication of this paper.

\section{Acknowledgment}

The authors are grateful to the Brazilian Agency FAPEAM (Fundação de Amparo à Pesquisa do Estado do Amazonas) for the financial support for this publication based on the Edital Papac 020/2013.

\section{References}

[1] S. T. Navale, A. T. Mane, A. A. Ghanwat, A. R. Mulik, and V. B. Patil, "Camphor sulfonic acid (CSA) doped polypyrrole (PPy) films: measurement of microstructural and optoelectronic properties," Measurement, vol. 50, no. 1, pp. 363-369, 2014.

[2] A. Riul Jr., A. M. G. Soto, S. V. Mello, S. Bone, D. M. Taylor, and L. H. C. Mattoso, "An electronic tongue using polypyrrole and polyaniline," Synthetic Metals, vol. 132, no. 2, pp. 109-116, 2003.

[3] D. Zhang, "Preparation of core-shell structured aluminapolyaniline particles and their application for corrosion protection," Journal of Applied Polymer Science, vol. 101, no. 6, pp. 4372-4377, 2006.

[4] J. H. Sung, S.-J. Kim, and K.-H. Lee, "Preparation of compact polyaniline films: electrochemical synthesis using agar gel template and charge-storage applications," Journal of Power Sources, vol. 126, no. 1-2, pp. 258-267, 2004. 
[5] T. Ahuja, I. A. Mir, D. Kumar, and Rajesh, "Biomolecular immobilization on conducting polymers for biosensing applications," Biomaterials, vol. 28, no. 5, pp. 791-805, 2007.

[6] C. K. Chiang, C. R. Fincher Jr., Y. W. Park et al., "Electrical conductivity in doped polyacetylene," Physical Review Letters, vol. 39, no. 17, pp. 1098-1101, 1977.

[7] R. Ansari, "Polypyrrole conducting electroactive polymers: synthesis and stability studies," E-Journal of Chemistry, vol. 3, no. 4, pp. 186-201, 2006.

[8] M. A. Chougule, S. G. Pawar, P. R. Godse, R. N. Mulik, S. Sen, and V. B. Patil, "Synthesis and characterization of polypyrrole (PPy) thin films," Soft Nanoscience Letters, vol. 01, no. 01, pp. 610, 2011.

[9] T. Patois, J.-B. Sanchez, F. Berger, J.-Y. Rauch, P. Fievet, and B. Lakard, "Ammonia gas sensors based on polypyrrole films: influence of electrodeposition parameters," Sensors and Actuators B: Chemical, vol. 171-172, pp. 431-439, 2012.

[10] Y. Liu, Y. Chu, and L. Yang, "Adjusting the inner-structure of polypyrrole nanoparticles through microemulsion polymerization," Materials Chemistry and Physics, vol. 98, no. 2-3, pp. 304308, 2006.

[11] M. R. Warren and J. D. Madden, "A structural, electronic and electrochemical study of polypyrrole as a function of oxidation state," Synthetic Metals, vol. 156, no. 9-10, pp. 724-730, 2006.

[12] J. D. W. Madden, N. A. Vandesteeg, P. A. Anquetil et al., "Artificial muscle technology: physical principles and naval prospects," IEEE Journal of Oceanic Engineering, vol. 29, no. 3, pp. 706-728, 2004.

[13] E. Smela, "Conjugated polymer actuators for biomedical applications," Advanced Materials, vol. 15, no. 6, pp. 481-494, 2003.

[14] T. F. Otero and M. Broschart, "Polypyrrole artificial muscles: a new rhombic element. Construction and electrochemomechanical characterization," Journal of Applied Electrochemistry, vol. 36, no. 2, pp. 205-214, 2006.

[15] G. M. Spinks, L. Liu, G. G. Wallace, and D. Zhou, "Strain response from polypyrrole actuators under load," Advanced Functional Materials, vol. 12, no. 6-7, pp. 437-440, 2002.

[16] Y. Yang, J. Liu, and M. Wan, "Self-assembled conducting polypyrrole micro/nanotubes," Nanotechnology, vol. 13, no. 6, pp. 771-773, 2002.

[17] I. Díez, K. Tauer, and B. Schulz, "Polypyrrole tubes via casting of pyrrole- $\beta$-naphthalenesulfonic acid rods," Colloid and Polymer Science, vol. 283, no. 2, pp. 125-132, 2004.

[18] I. Díez, F. Emmerling, F. Malz, C. Jäger, B. Schulz, and I. Orgzall, "Origin of templating processes in polypyrrole synthesis," Materials Chemistry and Physics, vol. 112, no. 1, pp. 154-161, 2008.

[19] G. B. Street, T. C. Clarke, R. H. Geiss et al., "Characterization of polypyrrole," Journal de Physique Colloques, vol. 44, no. 2, pp. C3-599-C3-606, 1983

[20] H. K. Chitte, N. V. Bhat, V. E. Walunj, and G. N. Shinde, "Synthesis of polypyrrole using ferric chloride $\left(\mathrm{FeCl}_{3}\right)$ as oxidant together with some dopants for use in gas sensors," Journal of Sensor Technology, vol. 1, pp. 47-56, 2011.

[21] J. Rodríguez-Carvajal, "Recent developments of the program FullProf," IUCr Commission on Powder Diffraction Newsletter, vol. 26, pp. 12-19, 2001.

[22] G. S. Pawley, "Unit-cell refinement from powder diffraction scans," Journal of Applied Crystallography, vol. 14, no. 6, pp. 357361, 1981.

[23] P. Thompson, D. E. Cox, and J. B. Hastings, "Rietveld refinement of debye-scherrer synchrotron $\mathrm{x}$-ray data from $\mathrm{Al}_{2} \mathrm{O}_{3}$," Journal of Applied Crystallography, vol. 20, no. 2, pp. 79-83, 1987.
[24] N. C. Popa, "The (hkl) Dependence of diffraction-line broadening caused by strain and size for all laue groups in Rietveld refinement," Journal of Applied Crystallography, vol. 31, no. 2, pp. 176-180, 1998.

[25] A. Le Bail, "New developments in microstructure analysis via rietveld refinement," Advances in X-Ray Analysis, vol. 42, pp. 191-203, 2000.

[26] J. Rodríguez-Carvajal and T. Roisnel, "Line broadening analysis using FULLPROF: determination of microstructural properties," Materials Science Forum, vol. 443-444, no. 2, pp. 123-126, 2004.

[27] P. Giannozzi, S. Baroni, N. Bonini et al., "QUANTUM ESPRESSO: a modular and open-source software project for quantum simulations of materials," Journal of Physics Condensed Matter, vol. 21, no. 39, Article ID 395502, 19 pages, 2009.

[28] M. C. Payne, M. P. Teter, D. C. Allan, T. A. Arias, and J. D. Joannopoulos, "Iterative minimization techniques for ab initio total-energy calculations: molecular dynamics and conjugate gradients," Reviews of Modern Physics, vol. 64, no. 4, pp. 10451097, 1992.

[29] A. Kokalj, "Computer graphics and graphical user interfaces as tools in simulations of matter at the atomic scale," Computational Materials Science, vol. 28, no. 2, pp. 155-168, 2003.

[30] R. M. Silverstein, F. X. Webster, and D. J. Kiemle, Spectrometric Identification of Organic Compounds, John Wiley \& Sons, New York, NY, USA, 7th edition, 2005.

[31] J. M. Hollas, Modern Spectroscopy, John Wiley \& Sons, London, UK, 4th edition, 2004.

[32] M. A. Chougule, S. G. Pawar, P. R. Godse, R. N. Mulik, S. Sen, and V. B. Patil, "Synthesis and characterization of polypyrrole (PPy) thin films," Soft Nanoscience Letters, vol. 1, no. 1, pp. 6-10, 2011.

[33] M. A. Chougule, S. Sen, and V. B. Patil, "Polypyrrole-ZnO hybrid sensor: effect of camphor sulfonic acid doping on physical and gas sensing properties," Synthetic Metals, vol. 162, no. 17-18, pp. 1598-1603, 2012.

[34] H. J. Kharat, K. P. Kakde, P. A. Savale, K. Datta, P. Ghosh, and M. D. Shirsat, "Synthesis of polypyrrole films for the development of ammonia sensor," Polymers for Advanced Technologies, vol. 18, no. 5, pp. 397-402, 2007.

[35] B. Tian and G. Zerbi, "Lattice dynamics and vibrational spectra of polypyrrole," The Journal of Chemical Physics, vol. 92, no. 6, pp. 3886-3891, 1990.

[36] M. A. Chougule, S. G. Pawar, P. R. Godse, R. N. Mulik, S. Sen, and V. B. Patil, "Synthesis and characterization of polypyrrole (ppy) thin films," Soft Nanoscience Letters, vol. 1, no. 1, pp. 6-10, 2011.

[37] T. K. Vishnuvardhan, V. R. Kulkarni, C. Basavaraja, and S. C. Raghavendra, "Synthesis, characterization and a.c. conductivity of polypyrrole $/ \mathrm{Y}_{2} \mathrm{O}_{3}$ composites," Bulletin of Materials Science, vol. 29, no. 1, pp. 77-83, 2006.

[38] J. P. Pouget, M. E. Jozefowicz, A. J. Epstein, X. Tang, and A. G. MacDiarmid, "X-ray structure of polyaniline," Macromolecules, vol. 24, no. 3, pp. 779-789, 1991.

[39] A. J. Epstein and A. G. Macdiarmid, "Structure, order and the metallic state in polyaniline and its derivatives," Synthetic Metals, vol. 41, no. 1-2, pp. 601-606, 1991.

[40] W. Feng, E. Sun, A. Fujii, H. Wu, K. Niihara, and K. Yoshino, "Synthesis and characterization of photoconducting polyaniline- $\mathrm{TiO}_{2}$ nanocomposite," Bulletin of the Chemical Society of Japan, vol. 73, no. 11, pp. 2627-2633, 2000. 
[41] L. Akcelrud, Fundamentos da ciência dos polímeros, Manole, Barueri, Brazil, 2007.

[42] S. V. Canevarolo Jr., Ciências dos Polímeros: Um Texto Básico para Tecnólogos e Engenheiros, Artliber, São Paulo, Brazil, 2002.

[43] N. Terinte, R. Ibbett, and K. C Schuster, "Overview on native cellulose and microcrystalline cellulose I structure studied by $\mathrm{X}$-ray diffraction (WADX): comparison between measurements technique," Lenzinger Berichte, vol. 89, pp. 118-131, 2011.

[44] J. Vlassenbroeck, H. Janssen, and R. Van Dooren, "A direct Chebyshev approach with practical applicability in optimal control problems," in Proceedings of the 8th IFAC Triennial World Congress, vol. 3, pp. 159-164, Kyoto, Japan, 1981.

[45] L. Manzato, D. M. Trichês, S. M. de Souza, and M. F. de Oliveira, "Synthesis of nanostructured $\mathrm{SnO}$ and $\mathrm{SnO}_{2}$ by high-energy milling of Sn powder with stearic acid," Journal of Materials Research, vol. 29, no. 1, pp. 84-89, 2014.

[46] S. J. Coles, P. A. Gale, and M. B. Hursthouse, Polypyrrole Hemikis (Tetramethylammonium Chloride), Cambridge Crystallographic Data Centre (CCDC), Cambridge, UK, 2002.

[47] A. Le Bail, "Whole powder pattern decomposition methods and applications: a retrospection," Powder Diffraction, vol. 20, no. 4, pp. 316-326, 2005.

[48] A. Le Bail, H. Duroy, and J. L. Fourquet, "Ab-initio structure determination of $\mathrm{LiSbWO}_{6}$ by X-ray powder diffraction," Materials Research Bulletin, vol. 23, no. 3, pp. 447-452, 1988.

[49] E. A. Sanches, L. C. A. Gomes, J. C. Soares, G. R. Da Silva, and Y. P. Mascarenhas, "Characterization of poly(o-methoxyaniline) Emeraldine-base form obtained at different time neutralization," Journal of Molecular Structure, vol. 1063, no. 1, pp. 336340, 2014.

[50] E. A. Sanches, J. C. Soares, A. C. Mafud, E. G. R. Fernandes, F. L. Leite, and Y. P. Mascarenhas, "Structural characterization of Chloride Salt of conducting polyaniline obtained by XRD, SAXD, SAXS and SEM," Journal of Molecular Structure, vol. 1036, pp. 121-126, 2013.

[51] A. de S. Silva, J. C. Soares, A. C. Mafud et al., "Structural and morphological characterization of Poly(o-ethoxyaniline) Emeraldine-salt form using FTIR, XRD, LeBail method and SEM," Journal of Molecular Structure, vol. 1071, pp. 1-5, 2014.

[52] J. Rodríguez-Carvajal, "IUCr commission on powder diffraction," Newsletter, vol. 26, p. 12, 2001.

[53] M. Casas-Cabanas, M. R. Palacín, and J. Rodríguez-Carvajal, "Microstructural analysis of nickel hydroxide: anisotropic size versus stacking faults," Powder Diffraction, vol. 20, no. 4, pp. 334-344, 2005.

[54] J. Gonzalez-Platas, J. Rodríguez-Carvajal, and G. Fourier, "A Windows/Linux program to calculate and display Fourier maps," FullProf Suite.

[55] J. C. Soares, A. Brisolari, V. D. C. Rodrigues, E. A. Sanches, and D. Gonçalves, "Amperometric urea biosensors based on the entrapment of urease in polypyrrole films," Reactive \& Functional Polymers, vol. 72, no. 2, pp. 148-152, 2012.

[56] A. S. Liu and M. A. S. Oliveira, "Corrosion control of aluminum surfaces by polypyrrole films: influence of electrolyte," Materials Research, vol. 10, no. 2, pp. 205-209, 2007.

[57] M. Bazzaoui, L. Martins, E. A. Bazzaoui, and J. I. Martins, "New single-step electrosynthesis process of homogeneous and strongly adherent polypyrrole films on iron electrodes in aqueous medium," Electrochimica Acta, vol. 47, no. 18, pp. $2953-$ 2962, 2002.
[58] N. P. S. Chauhan, R. Ameta, R. Ameta, and S. C. Ameta, "Thermal and conducting behaviour of emeraldine base (EB) form of polyaniline (PANI)," Indian Journal of Chemical Technology, vol. 18 , no. 2, pp. 118-122, 2011. 

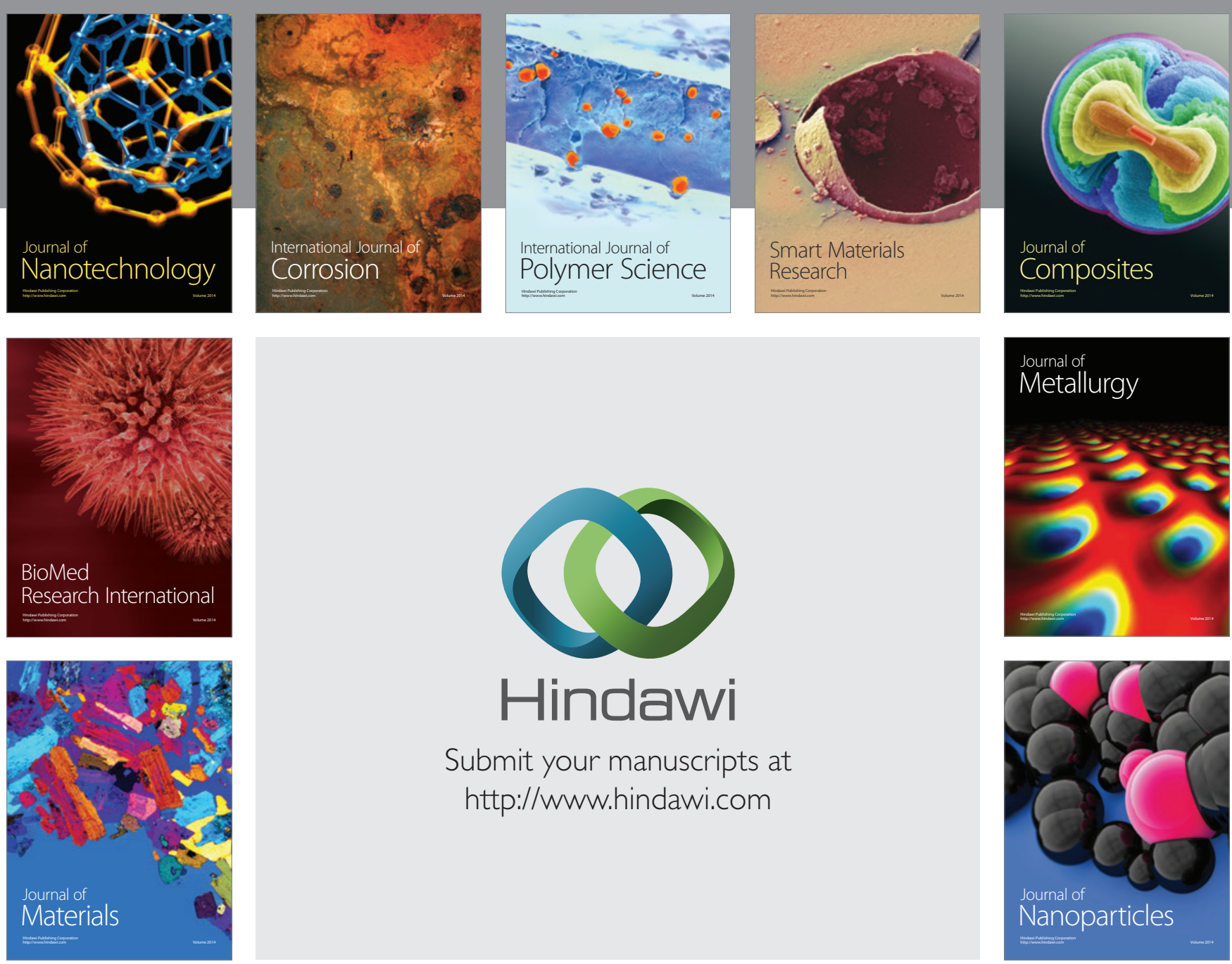

Submit your manuscripts at http://www.hindawi.com
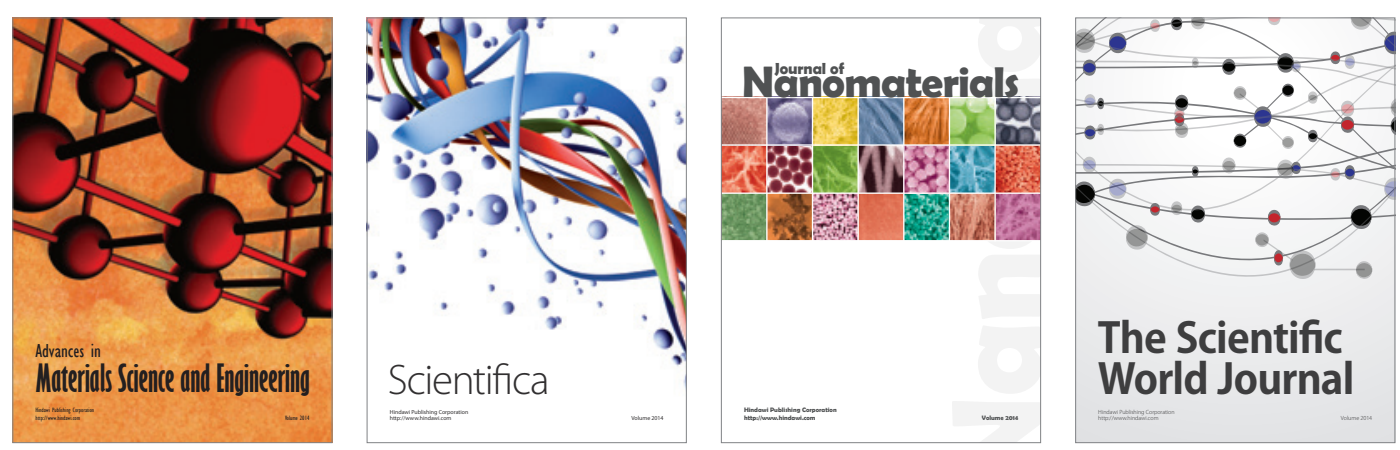

\section{The Scientific World Journal}
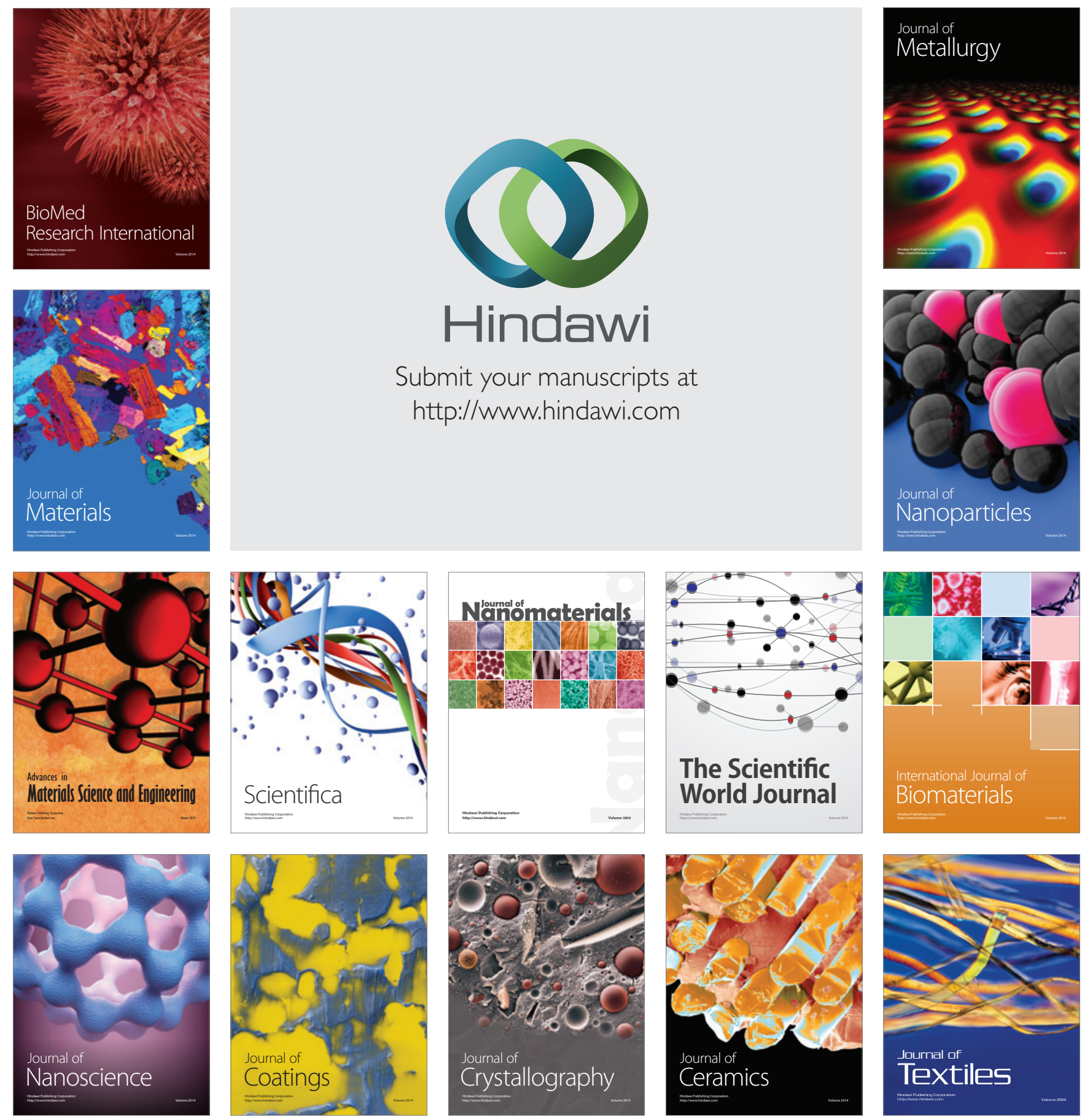